\title{
Correction to: Safety, Tolerability and Pharmacokinetics of Single Dose Polyethylene Glycolated Exenatide Injection (PB-119) in Healthy Volunteers
}

\author{
Hong Cui ${ }^{1}$ Cai-Yun Zhao ${ }^{1} \cdot$ Yuan Lv $^{1} \cdot$ Min-Ji Wei ${ }^{1} \cdot$ Yan Zhu ${ }^{1} \cdot$ Yun Li ${ }^{1} \cdot$ Ya-Hong Xia ${ }^{1} \cdot$ Yan Liu ${ }^{1} \cdot$ Ji-Hong Tian $^{1}$. \\ Pu Zhang ${ }^{1}$
}

Published online: 6 April 2020

(c) Springer Nature Switzerland AG 2020

\section{Correction to: \\ European Journal of Drug Metabolism and \\ Pharmacokinetics \\ https://doi.org/10.1007/s13318-020-00605-9}

The original version of this article unfortunately contained few errors. Funding section, which previously read:

No funding was received to conduct this study.

Should read:

This study was funded by PegBio Co., Ltd.

Last paragraph of "Introduction" line 2 should read as “.....developed by Peg Biomedical........."

The original article can be found online at https://doi.org/10.1007/ s13318-020-00605-9.

Yuan Lv

LY52850@163.com

1 Institute of Clinical Pharmacology, Peking University First Hospital, No. 8 Xishiku Street, Xicheng District, Beijing 100034, China 